\title{
Comparação entre os métodos
subjetivo e objetivo para avaliação da dor em idosos
}

Comparison between subjective

and objective methods for pain

FisiSenectus. Unochapecó Ano 5, n. 1 - Jan/Jun. 2017 assessment in elderly p. $13-20$

Bruna da Fonseca. bruna_1909@unochapeco.edu.br Acadêmica do Curso de Medicina da Universidade Comunitária da Região de Chapecó - UNOCHAPECÓ.

Bruna Paola Defáveri. brunapaola@unochapeco.edu.br Acadêmica do Curso de Medicina da Universidade Comunitária da Região de Chapecó - UNOCHAPECÓ.

Paula Zeni. paulazeni@unochapeco.edu.br

Fisioterapeuta. Mestre em Saúde e Doutora em Fisiologia. Docente da Área de Ciências da Saúde - UNOCHAPECÓ.

\section{Resumo}

Objetivo: Comparar a dor em idosos avaliadas por método subjetivo e quantificadas por método objetivo e também avaliar a eficácia do algômetro na mensuração da dor. Metodologia: Estudo quantitativo transversal, do tipo quase experimental, com amostra de 58 idosos. A coleta de dados foi realizada através de questionário sociodemográfico, escala numérica (EN), escala visual analógica (EVA) e algometria. Resultados: 0 principal local de queixa de dor foi a coluna lombar e os locais com maior dor por pressão foram o quadrante lateral inferior do glúteo esquerdo nas mulheres e face anterior medial do joelho esquerdo nos homens. Não houve correlação entre a algometria e as escalas, porém houve correlação significativa entre as duas escalas. Discussão: Outras pesquisas também apresentaram a coluna lombar como local de maior dor e as mulheres com maior queixa álgica, possivelmente por fatores biopsicológicos do sexo feminino. Há poucos estudos sobre o uso do algômetro; entre os encontrados houve confirmação de que o envelhecimento altera a percepção de dor por pressão e que o algômetro e as escalas subjetivas não tiveram correlação. 0 uso de escalas apresentou-se como uma forma eficaz de avaliação da dor pela sua praticidade. Conclusão: A queixa álgica foi maior na coluna lombar e as mulheres apresentaram maior percepção de dor. Não houve correlação entre os métodos algometria e escalas, sugerindo que a algometria não foi eficaz como forma de avaliação de dor em idosos.

\section{Palavras-chave}

Idosos; Dor; Medição da dor.

\section{Fisiß̊nectus}




\begin{abstract}
Objective: Comparing pain in the elderly evaluated by subjective method and quantified by objective method and also to evaluate the effectiveness of the algometer in the measurement of pain. Methodology: A quasiexperimental cross-sectional quantitative study with a sample of 58 elderly subjects. Data were collected through a sociodemographic questionnaire, numerical scale (EN), visual analogue scale (EVA) and algometry. Results: The main site of complaint of pain was the lumbar spine and the sites with the greatest pressure pain were the lower lateral quadrant of the left gluteus in the females and the medial anterior aspect of the left knee in the men. There was no correlation between algometry and scales, but there was a significant correlation between the two scales. Discussion: Other studies also presented the lumbar spine as the site of greatest pain and the women with the most painful complaints, possibly due to female biopsychological factors. There are few studies on the use of algometer; Among those found there was confirmation that aging alters the perception of pressure pain and that the algometer and the subjective scales had no correlation. The use of scales was presented as an effective way of assessing pain for its practicality. Conclusions: The pain complaint was higher in the lumbar spine and the women presented greater pain perception. There was no correlation between algometry and scaling methods, suggesting that algometry was not effective as a form of pain evaluation in the elderly.
\end{abstract}

\title{
Keywords
}

Elderly; Ache; Pain measurement.

\section{Introdução}

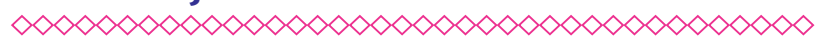

A dor é um sinal clínico comum na população idosa, muitas vezes subestimada e subtratada pelos profissionais da saúde. Quando persistente, a dor pode incapacitar e comprometer a qualidade de vida do geronte ${ }^{1}$.

Há poucos instrumentos de mensuração da dor específicos para a população idosa devido à dificuldade desta faixa etária em compreender o que é proposto através dos testes e expressar de forma fidedigna a intensidade álgica. Assim, percebe-se a necessidade de haver um método de estudo que mensure a dor de forma objetiva, por isso a relevância de se aplicar o uso do algômetro e comparar seus resultados com os dados subjetivos coletados ${ }^{2}$.

Diante disso, esta pesquisa buscou comparar as respostas de dor em idosos avaliadas pelo método subjetivo e quantificadas pelo método objetivo, além de avaliar a eficácia do uso de um aparelho (algômetro) na mensuração da dor e analisar a percepção de dor em indivíduos idosos, considerando as particularidades do processo de envelhecimento.

\section{Materiais e métodos}

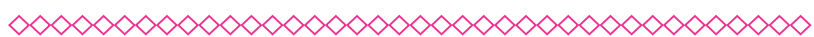

0 estudo foi quantitativo transversal, do tipo quase experimental. A amostra foi composta por 58 idosos que passaram por atendimento médico em um centro de saúde no oeste catarinense durante o período de coleta de dados, que tinham 60 anos de idade ou mais, apresentaram queixa álgica (identificada pelo questionário sociodemográfico) e aceitaram participar da pesquisa através da assinatura do termo de consentimento livre esclarecido (TCLE). Os critérios de exclusão foram: ter deficiência cognitiva e/ou auditiva que impediam de responder os questionários utilizados. Durante a coleta de dados, os voluntários foram caracterizados enquanto perfil sociodemográfico pelo questionário sociodemográfico. Em seguida, foram avaliados em relação à percepção de dor utilizando o instrumento escala visual analógica (EVA), que consiste em uma linha reta de dez centímetros entre duas extremidades, tendo à esquerda um ponto nomeado como "sem dor" e a direita outro ponto que indica a "pior dor imaginável". Na linha descrita o participante da amostra marcou o ponto que melhor equivaleu a sua dor. A distância encontrada do ponto "sem dor" até o ponto marcado 
pelo participante foi medida e posteriormente comparada a outros métodos ${ }^{3}$.

Também foram avaliados pela escala numérica (EN), sendo esta semelhante à EVA. Esse instrumento consiste em uma reta com onze marcações numeradas de zero a dez, ou seja, cada número representa uma quantidade de dor. Na régua descrita o participante da amostra aponta um número de zero a dez que equivaleria à intensidade da sua dor. 0 número obtido foi registrado e posteriormente comparado a outros métodos ${ }^{4}$.

Por fim, foi aplicada a avaliação de dor utilizando o algômetro, (DDK 10 kgf-Kratos $\left.{ }^{\circledR}\right) .0$ algômetro é um aparelho capaz de mensurar a dor de maneira objetiva, quantificando-a através de uma pressão contínua que aumentará até o momento em que o participante da amostra suporte tal pressão, sendo esse o seu limite. 0 exame com o algômetro aconteceu da seguinte forma: (1) a algometria foi aplicada em todos os locais selecionados; (2) o exame foi repetido dois minutos depois, nos mesmos locais; (3) foi feita uma média aritmética entre os dois valores obtidos em cada local; (4) cada valor resultante das médias foi considerado como o limite de dor suportado pelo participante naquele local ${ }^{3}$. Destacamos que o método de aferição e quantificação de dor por meio do algômetro não causou qualquer prejuízo ou dano físico aos voluntários.

Os locais anatômicos avaliados foram:

- tubérculo maior do úmero;

- espinha ilíaca póstero-superior;

- quadrante lateral inferior do glúteo;

- face posterior da cabeça da fíbula;

- face anterior medial do joelho.

Todos os pontos foram avaliados bilateralmente.

Todos os voluntários receberam o Termo de Consentimento Livre e Esclarecido (TCLE) no qual havia a explicação dos procedimentos utilizados, as recomendações para o dia da coleta, bem como objetivos e as justificativas para a realização do estudo, os riscos e benefícios aos quais estariam expostos, e demais itens descritos nas Diretrizes do Conselho Nacional de Saúde (Resolução 466/12).

Foi garantido ao voluntário o direito de se recusar em participar do estudo em qualquer momento, sem nenhum prejuízo, e the foi informado o telefone dos pesquisadores para a solução de quaisquer dúvidas que pudessem surgir posteriormente. Após a leitura do documento, foram esclarecidas todas as dúvidas dos voluntários, que posteriormente assinaram duas vias do TCLE. 0 voluntário recebeu uma das vias e a outra foi arquivada pelos pesquisadores.

O projeto foi submetido ao Comitê de Ética e Pesquisa, sendo aprovado pelo mesmo no dia 31 de agosto de 2015. O número do protocolo de aprovação é 1.207.450.

A análise dos dados foi realizada com o programa estatístico Statistical Package for the Social Sciences (IBM - SPSS) para Windows, versão 21,0. 0 teste utilizado na análise estatística foi o coeficiente de correlação de Pearson ( $r$ ) para determinar a força da correlação entre intensidade de dor e o valor de limiar de dor à pressão.

\section{Resultados}

$\infty \infty \infty \times \infty \times \infty \times \infty \times \infty \times \infty \times \infty \times \infty \times \infty \times \infty \times \infty \infty$

A coleta de dados foi iniciada no dia 21 de outubro de 2015 e foi finalizada no dia 05 de julho de 2016, totalizando 58 voluntários. 0 questionário sociodemográfico aplicado destacou dados referentes às características da amostra quanto ao gênero, idade, sensação de dor, local da dor e medicamentos utilizados.

A amostra foi composta de 17 homens (29,3\%) e 41 mulheres (70,6\%), com idade média de 69,06 (DP 5,79) e 68,49 (DP 5,94), respectivamente. Os principais locais que apresentaram queixa álgica foram coluna lombar em ambos os sexos $(55,89 \%)$ entre as mulheres e $58,8 \%$ entre os homens), seguido de articulações no sexo feminino (34,02\%) e membros inferiores no masculino (58,8\%). 0 uso de anti-inflamatórios não esteroides para o alívio da dor destacou-se entre as mulheres (41,31\%), já os homens referiram uso mais frequente de analgésicos orais (41,16\%). Quanto a presença de doenças, hipertensão arterial sistêmica esteve presente em parcela significativa da amostra $(68,04 \%$ das mulheres, 52,92\% dos homens), onde também se destacou a osteoporose em mulheres (38,88\%) e a depressão em ambos (12,15\% das mulheres, $11,76 \%$ dos homens). 
$\mathrm{Na}$ avaliação da dor através das escalas subjetivas, a EVA apresentou média 6,68 (DP 2,97) para as mulheres e 4,71 (DP 3,18) para os homens. Já a EN teve média 6,92 (DP 2,38) para as mulheres e 5,14 (DP 3,05) para os homens.

$\mathrm{Na}$ avaliação através do método objetivo algômetro, os locais que apresentaram os menores valores para percepção de dor foram quadrante lateral inferior do glúteo direito (média 2,41, DP 1,29) juntamente com face anterior medial do joelho esquerdo (média 2,41, DP 1,42) entre as mulheres, e face anterior medial do joelho esquerdo (média 2,73, DP 1,38 ) entre os homens.

A correlação entre dor percebida e dor por pressão, ou seja, entre a dor avaliada pelos os instrumentos EVA, EN e algometria está apresentada na Tabela 1.

\section{Discussão}

$\infty \times \infty \times \infty \times \infty \times \infty \times \infty \times \infty \times \infty \times \infty \times \infty \times \infty \times \infty \times \infty$

Os locais que apresentaram mais queixas de dor entre os participantes da amostra foram coluna lombar em ambos os sexos seguida de articulações na população feminina e membros inferiores na população masculina. Um estudo realizado na cidade de São Paulo, envolvendo 377 idosos, apresentou como locais de maior dor referida a região lombar e membros inferiores 5 . Outro estudo feito com uma amostra de 451 idosos na cidade de Londrina (PR) apontou como os principais locais de dor em idosos a região lombar e membros inferiores ${ }^{6}$.

Uma pesquisa realizada nos Estados Unidos da América, com uma amostra de 27.035 pessoas a partir de 18 anos de idade também teve a coluna lombar como o local com mais queixas álgicas e maiores índices de dor foram diretamente proporcionais a idades mais avançadas. Estão também entre os locais com mais queixa álgica as articulações (joelhos, ombros, pés) e membros inferiores. As mulheres nesse estudo apresentaram mais dor que os homens ${ }^{7}$.

Os estudos corroboram os dados apresentados nesta pesquisa, que identificou maior dor na coluna lombar referida através do questionário sociodemográfico e também apresentou menor limiar de dor através de algometria em membros inferiores (quadrante lateral inferior do glúteo esquerdo nas mulheres e face anterior medial do joelho esquerdo nos homens). Esse fato possivelmente tem relação com o processo de envelhecimento e suas mudanças, que vão desde alterações fisiológicas na constituição musculoesquelética, como perda de massa muscular, atrofia e enfraquecimento ósseo, até o aparecimento de doenças crônicas e traumas comuns à idade, como osteoporose e fratura de fêmur ${ }^{8,9}$.

A dor foi maior nas mulheres em todos os métodos utilizados neste estudo (algometria, EVA, EN e questionário sociodemográfico). Diversos estudos, com diferentes amostras e metodologias, confirmam estes resultados. Um deles foi composto por uma amostra de 1705 idosos e buscou estimar a prevalência de dor nessa população da cidade de Florianópolis (SC), o qual apresentou prevalência de $82 \%$ de dor crônica nas mulheres em relação aos homens ${ }^{10}$. Outro estudo realizado por Pereira e colaboradores ${ }^{11}$ utilizou a EN para avaliar a dor em uma população de 934 idosos e também destacou maior prevalência de dor nas mulheres. No estudo de Johannes e colaboradores ${ }^{7}$, a prevalência de dor entre as mulheres foi $34,3 \%$, maior do que para os homens $(26,7 \%)$.

Possiveis explicações foram abordadas pela literatura no que tange a maior referência de dor no sexo feminino: entre os fatores estão as características pessoais, como as funções biológicas e psicológicas, influenciadas por fatores hormonais, como alterações nos níveis de estrogênio, que quando reduzidos podem levar a desordens neuromusculares e também causar labilidade emocional, uma vez que o estrogênio auxilia na estimulação dos sistemas adrenérgico e serotoninérgico, os quais influenciam o estado de bem-estar. Estes fatores somam-se à dupla jornada de trabalho (emprego e família) e também ao fato de que as mulheres relatam mais dor do que os homens, já que estes são socialmente menos propensos a demonstrarem suas fragilidades ${ }^{10-12}$.

A dor nas mulheres pode ainda ser causa ou consequência de transtornos depressivos, os quais foram referidos em $12,15 \%$ das voluntárias desta pesquisa. As alterações de humor, como a depressão, podem ser consequências do período de climatério e menopausa que faz parte do 
envelhecimento feminino e que leva à deficiência estrogênica, a qual pode influenciar na secreção de neurotransmissores relacionados à depressão ${ }^{12}$.

São poucos os estudos relativos ao uso do aparelho algômetro. Entre os encontrados está uma pesquisa realizada na Universidade de São Paulo com amostra de 210 pessoas, que avaliou o limiar de dor à pressão na articulação temporomandibular com algometria e EVA, metodologia similar a este estudo, concluindo que há uma fraca, porém significante correlação entre a intensidade da dor referida e o limiar de dor com o aparelho ${ }^{13}$. Outra pesquisa que utilizou algometria como forma de avaliação de dor comparada às escalas numérica e visual analógica apresentou correlação moderada e estatisticamente significativa ${ }^{14}$.

Um estudo buscou comparar os limiares de dor por pressão através de testes com a algometria em músculos da cabeça e pescoço, com uma amostra de 40 indivíduos sendo 20 jovens, e 20 idosos. Diante dos resultados, houve uma diferença significativa entre os limiares de dor por pressão, sendo mais elevados nos idosos em comparação aos jovens. Os dados mostraram que os limiares de dor por pressão podem aumentar diante do processo de envelhecimento nos músculos analisados; destacaram também que o maior limiar de dor não significa que os idosos sintam, necessariamente, menos dor que os jovens, mas levanta a possibilidade de haver um atraso na percepção da dor (pela possível desmielinização neuronal) ${ }^{15}$.

0 possível aumento do limiar de dor por pressão nos idosos pode ser explicado pelo próprio processo fisiológico do envelhecimento, onde há diminuição da massa muscular esquelética e consequente diminuição do tônus muscular, e ainda alterações na percepção, transmissão e processamento da dor ${ }^{5,15,16}$

Por outro lado, em um estudo que utilizou métodos semelhantes a esta pesquisa, porém, com amostra composta por um grupo de idosos e outro grupo de jovens, a comparação entre algometria e EVA e entre os grupos e concluiu que não houve diferenças significativas entre a percepção de dor entre idosos e jovens. Além disso, os resultados não demonstraram correlação entre algometria e EVA, corroborando nossos resultados ${ }^{17}$.
Este estudo não apresentou significância estatística na correlação entre algometria e escalas de dor, o que também foi constatado no estudo de Piovesan e colaboradores ${ }^{18}$, que concluiu que a algometria, apesar de ser um método objetivo, apresenta limitações, como variação entre aferições no mesmo indivíduo em períodos diferentes, variações essas que, conforme observado nesta pesquisa, podem ser influenciadas por diversos fatores, como ansiedade, tensão no momento da coleta e locais testados que não coincidiram com os locais referidos pelos voluntários.

Além disso, ao comparar a algometria com uso de escalas percebe-se maior dificuldade por parte da amostra em compreender as etapas do processo de algometria, por exemplo, o momento exato para indicar o início de percepção da dor. Outra limitação deste estudo é o caráter multifatorial da dor, pois a dor percebida por algometria é uma dor por pressão provocada, diferente da dor física de origem patológica ou até mesmo influenciada por fatores emocionais.

As escalas, EN e EVA, se mostraram muito eficazes na avaliação da dor, apresentando significativa correlação entre elas. Portanto ainda são métodos aplicáveis para uma rápida e indispensável avaliação de dor. Entre as duas escalas, a EN é a mais recomendada devido à facilidade de uso, formato padronizado e fácil entendimento ${ }^{14}$.

Uma revisão de literatura que avaliou estudos sobre o uso de escalas de dor constatou que a escala preferida pelos pacientes, a qual demonstrou ser de aplicação mais fácil e confiável, foi a EN. A EVA se mostrou mais complicada e com maior risco de erro, principalmente em idosos ou com alguma alteração cognitiva. A maioria dos estudos revisados relatou uma boa correlação entre as escalas e que elas tendem a medir variações na mesma direção ${ }^{19}$.

Há outros métodos que também podem avaliar a dor, como o questionário de dor McGill, o qual possibilita uma caracterização ampla da dor e avalia a dor através de diversos descritores verbais, todavia apresenta limitações, pois sua eficácia depende de uma boa capacidade de comunicação e compreensão por parte do indivíduo avaliado e ainda é um método que demanda tempo para ser aplicado $^{20,21}$. 
Um estudo realizado com 101 idosos institucionalizados comparou a EN com a Escala de Descritores Verbais (EDV), uma escala de mensuração de dor que utiliza cinco descritores para determinar a intensidade da dor; todos os participantes da amostra foram capazes de julgar a dor pela EDV, $58,5 \%$ preferiram esta escala e as duas escalas tiveram correlação significativa, portanto a EDV também pode ser uma opção na mensuração da dor, ainda que de forma subjetiva ${ }^{22}$.

\section{Conclusão}

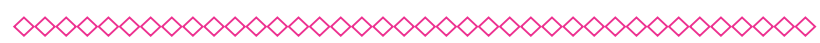

A dor foi avaliada pelos métodos algometria, escala visual analógica e escala numérica, sendo que a correlação não foi observada entre os métodos algometria e escalas, sugerindo que a algometria não foi eficaz na avaliação da dor em idosos, no entanto houve correlação forte entre os dados das escalas. A dor provocada por pressão foi maior no ponto face anterior medial do joelho esquerdo para homens e para as mulheres o quadrante lateral inferior esquerdo. Já o principal local de dor relatado por questionário foi a coluna lombar, refletindo a degeneração musculoesquelética do processo de envelhecimento. Quanto à percepção de dor, mulheres apresentaram maiores índices em relação aos homens.

Declaramos que este artigo é parte de monografia apresentada ao Curso de Graduação em Medicina da Universidade Comunitária da Região de Chapecó (UNOCHAPECÓ).

\section{Referências}

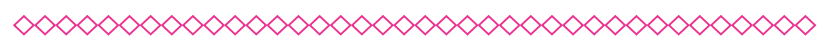

1. Gomes JCP, Teixeira MJ. Dor no idoso.

Moreira Jr Editora [Internet]. 2006 [acesso em 2015 mar 17]; 554-563. Disponível em: http://www.moreirajr.com.br/revistas.asp?id_ materia $=3465 \&$ fase $=$ imprime .

2. Andrade FA, Pereira LV, Sousa FAEF. Mensuração da dor no idoso: uma revisão. Rev Latino-Am Enfermagem $2006 \mathrm{Abr} ; 14(2): 271-276$.
3. Ferreira, APL. Efeito imediato da tens na dor, na função muscular e sua correlação com qualidade do sono e grau de catastrofização em indivíduos com disfunção temporomandibular [Tese]. Aracaju: Universidade Federal de Sergipe; 2014.

4. Polleto PR, Coury HJCG, Walsh IAP, MattieloRosa SM. Correlação entre métodos de autorelato e testes provocativos de avaliação da dor em indivíduos portadores de distúrbios osteomusculares relacionados ao trabalho. Rev Bras Fisioter 2004 Jul;8(3):223-229.

5. Dellaroza MSG, Pimenta CAM, Lebrão ML, Duarte YA. Associação de dor crônica com uso de serviços de saúde em idosos residentes em São Paulo. Rev Saúde Pública 2013 Jun;47(5):914-922.

6. Dellaroza MSG, Pimenta CAM, Matsuo T. Prevalência e caracterização da dor crônica em idosos não institucionalizados. Cad Saúde Pública 2007 Mai;23(5):1151-1160.

7. Johannes CB, Le TK, Zhou X, Johnston JA, Dworkin $\mathrm{RH}$. The Prevalence of Chronic Pain in United States Adults: Results of an InternetBased Survey. The Journal Of Pain 2010 Nov;11(11):1230-1239.

8. França DM, Senna-Fernandes V, Aguiar C, Amaral G, Oliveira R Cortez C, et al. Acupuntura na reabilitação da terceira idade. Fisioter Bras 2006 Dez;7(6):433-439.

9. Soares DS, Mello LM, Silva AS, Martinez EZ, Nunes AA. Fraturas de fêmur em idosos no Brasil: análise espaço-temporal de 2008 a 2012. Cad Saúde Pública 2014 Dez;30(12):2669-2678.

10. Santos FAA, Souza JB, Antes DL, d'Orsi E. Prevalência de dor crônica e sua associação com a situação sociodemográfica e atividade física no lazer em idosos de Florianópolis, Santa Catarina: estudo de base populacional. Rev Bras Epidemiol 2015 Mar;18(1):234-247.

11. Pereira LV, Vasconcelos PP, Souza LAF, Pereira GA, Nakatani AYK, Bachion MM. Prevalência, intensidade de dor crônica e autopercepção de saúde entre idosos: estudo de base populacional. Rev Latino-Am Enfermagem 2014 Jul;22(4): 662-669. 
12. Freitas F, Menke CH, Rivoire WA, Passos EP. Rotinas em Ginecologia. 6.ed. Porto Alegre: Artmed; 2011.

13.Stuginski-Barbosa J, Silva RS, Cunha CO, Bonjardim LR, Conti ACCF, Conti PCR. Pressure pain threshold and pain perception in temporomandibular disorder patients: is there any correlation?. Rev Dor 2015 Mar; 16(1):22-26.

14. Alfonsin MM. Correlação da algometria, escala análogo visual, escala numérica de avaliação da dor em mulheres com dor pélvica crônica [Dissertação]. Porto Alegre: Universidade Federal do Rio Grande do Sul; 2013.

15. Marini I, Bortolotti F, Borlolucci, ML, Inelmen EN, Gatto MR, Bonetti GA. Aging effect on pressure pain thresholds of head and neck muscles. Aging Clin Exp Res 2012 Mar;24(3): 239-244.

16. Arking R. Biologia do envelhecimento. 2.ed. Ribeirão Preto: Funpec; 2008.

17. Mesci E, Içagasioglu A, Atlig RS, Toslali S. Pain sensitivity in the elderly. Turk Geriatri Dergisi 2015 Jul;18(2):130-135.

18. Piovesan EJ, Tatsui CE, Kowacs PA, Lange MC, Pacheco C, Werneck LC. Utilização da algometria de pressão na determinação dos limiares de percepção dolorosa trigeminal em voluntários sadios: um novo protocolo de estudos. Arq. Neuro-psiquiatr 2001 Mar;59(1):92-96.

19. Hjermstad MJ, Fayers PM, Haugen DF, Caraceni A, Hanks GW, Loge JH, et al. Studies Comparing Numerical Rating Scales, Verbal Rating Scales, and Visual Analogue Scales for Assessment of Pain Intensity in Adults: A Systematic Literature Review. Journal Of Pain And Symptom Managemen 2011 Jun;41(6):1073-1093.

20. Silva MCOS, Silva PAB, Silva LB, Soares SM. Instrumentos de avaliação da dor crônica em idosos e suas implicações para a enfermagem. $\mathrm{R}$ Enferm Cent 0 Min 2011 Dez;4(1):560-570.

21. Melzack, R. The McGill Pain Questionnaire: major properties and scoring methods. Pain 1975 Set;1(3).

22.Pereira LV, Pereira GA, Moura LA, Fernandes RR. Intensidade da dor em idosos institucionalizados: comparação entre as escalas numérica e de descritores verbais. Rev Esc Enferm USP 2015 Out;49(5):804-810. 


\section{Anexos}

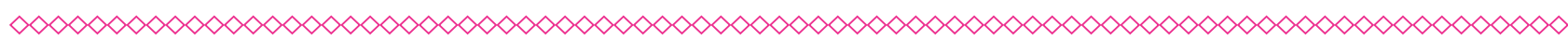

Tabela 1 - Correlação entre as escalas de avaliação da dor e algometria

\begin{tabular}{|c|c|c|c|c|}
\hline & & $\begin{array}{c}\text { Escala Visual } \\
\text { Analógica (EVA) }\end{array}$ & $\begin{array}{l}\text { Escala Numérica } \\
\text { (EN) }\end{array}$ & Algometria \\
\hline \multirow{2}{*}{$\begin{array}{l}\text { Escala Visual } \\
\text { Analógica } \\
\text { (EVA) }\end{array}$} & $\begin{array}{l}\text { Correlação de } \\
\text { Pearson }\end{array}$ & 1 & 0,770 & 0,135 \\
\hline & $\mathrm{N}$ & 55 & 55 & 55 \\
\hline \multirow{2}{*}{$\begin{array}{c}\text { Escala } \\
\text { Numérica (EN) }\end{array}$} & $\begin{array}{l}\text { Correlação de } \\
\text { Pearson }\end{array}$ & 0,770 & 1 & 0,020 \\
\hline & $\mathrm{N}$ & 55 & 55 & 55 \\
\hline \multirow[t]{2}{*}{ Algometria } & $\begin{array}{l}\text { Correlação de } \\
\text { Pearson }\end{array}$ & 0,135 & 0,020 & 1 \\
\hline & $\mathrm{N}$ & 55 & 55 & 58 \\
\hline
\end{tabular}

(clique para voltar ao texto) 